\title{
The use of indocyanine green in bariatric surgery: A systematic review
}

\author{
Francesco Maria Carrano $^{1}$, Nicola Di Lorenzo ${ }^{1 *}$ \\ ${ }^{1} \mathrm{PhD}$ in Applied Medical-Surgical Sciences, University of Rome "Tor Vergata", 00133 Rome (RM), Italy
}

To Cite

Carrano FM, Di Lorenzo N. The use of indocyanine green in bariatric surgery: A systematic review. J Gastric Surg 2020; 2(2): $41-44$

\section{Publication history}

Received: May 19, 2020

Accepted: May 22, 2020

Article in press: June 02, 2020

Published on line: June XX, 2020

\section{*Correspondence to}

Prof. Nicola Di Lorenzo

Department of Surgical Sciences

University of Rome "Tor Vergata"

Viale Oxford 81

00133 Rome (RM), Italy

nicola.di.lorenzo@uniroma2.it

Telephone: +390620903854

\begin{abstract}
Fluorescence-guided surgery is a recently developed technique in minimally invasive surgery in which a fluorescent dye is used to complement the surgeon's judgment in making real-time intraoperative assessment of organ vascularization and proper tissue perfusion. This technique has been adopted in several different surgical subspecialties with positive results, particularly in hepatobiliary and colorectal surgery. More recently, it has also been applied in bariatric surgery, with the aim of reducing the incidence of leaks. This paper reviews the relevant literature on the topic.
\end{abstract}

Keywords:

ICG, bariatric surgery, indocyanine green, fluorescence. 


\section{Introduction:}

The alarming prevalence of obesity, with its related burden of metabolic consequences, is a well-known problem. Metabolic surgery has been recognized as the most effective treatment for achieving significant weight loss as well as tangible improvements in glycemic control and a reduction of overall cardiovascular risk. [1] Surgery for obesity is also recognized as a safe treatment, with mortality rates comparable to those of other common procedures (e.g., appendectomy, cholecystectomy, hysterectomy), ranging from $0.3 \%$ to $2 \%$.[2] The occurrence of major adverse events at 30 days vary from $5.0 \%$ for Roux-en-Y gastric bypass to $2.6 \%$ for sleeve gastrectomy.[3] Technological improvements as well as the institution of accredited bariatric surgery programs are two of the main factors contributing to these favorable results.[4] One recently introduced technique in the field of bariatric surgery is indocyanine green (ICG) fluorescence angiography (ICG-FA). Indocyanine green, a fluorescent dye approved by the FDA and EMA for use in medical diagnostics, has been routinely used for years in ophthalmology, but recently several other fields of application have also been described in the literature, most notably colorectal, hepatobiliary, and oncologic surgery.[5] ICG absorbs near-infrared light at wavelengths between 800 to $810 \mathrm{~nm}$ and becomes fluorescent at $830 \mathrm{~nm}$, when excited by a laser source or by a near-infrared light at $820 \mathrm{~nm}$ wavelength.[6] Fluorescence can be visualized by special laparoscopic cameras and rendered on screen in several different colors, depending on the proprietary filters applied by each camera maker. Our paper reviews the literature on application of ICG in obesity surgery.

\section{Methods:}

This systematic review was performed in accordance with the PRISMA guidelines.[7] The study population included patients undergoing any bariatric surgical operation. The intervention object of the analysis was intraoperative ICG-FA assessment of vascularization. Any comparison was considered. Outcome: postoperative complications. The following exclusion criteria were selected: preclinical studies, non-bariatric surgical procedures, surgeries on obese patients performed for other reasons other than weight loss, and language other than English. Duplicate studies were removed. A systematic search of the literature (Figure 1) was undertaken by the two authors in PubMed, Google Scholar, and Embase, current through May 5, 2020, and included additional papers identified from the references. Search terms were "indocyanine green", "ICG", "fluorescence angiography", "ICG AND bariatric surgery", "ICG AND obesity", "ICG AND leak", and "dehiscence". The following data were extracted from each study: age, preoperative BMI, length of stay, and postoperative complications.[8]

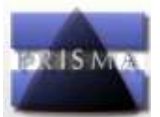

\section{PRISMA 2009 Flow Diagram}
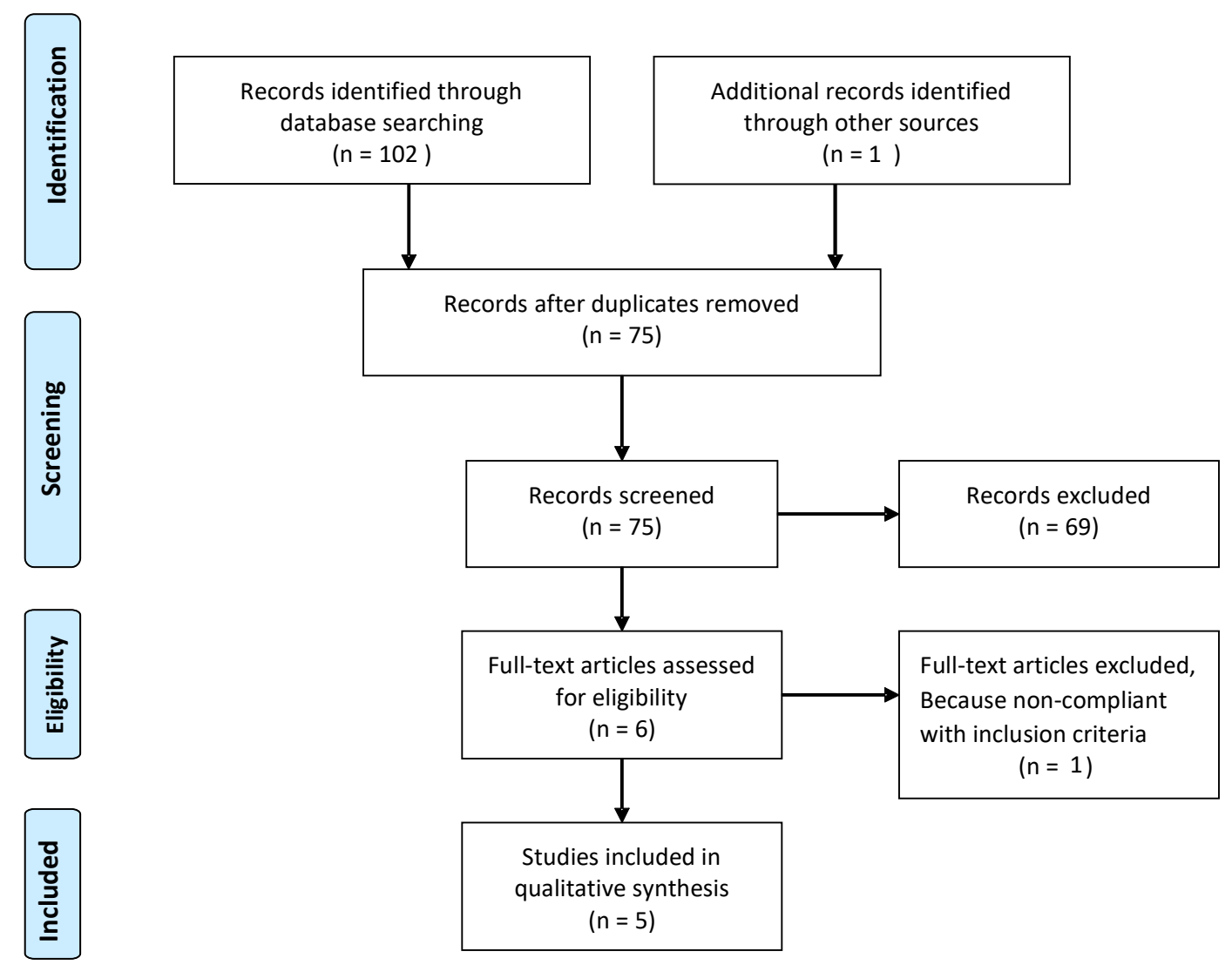

Figure 1: Flowchart of the records selection process. 


\begin{tabular}{|c|c|c|c|c|c|}
\hline Authors & $\begin{array}{l}\text { Patients } \\
\text { (N) }\end{array}$ & $\begin{array}{c}\text { Age } \\
\text { (mean } \pm \text { std dev) }\end{array}$ & $\begin{array}{c}\text { pre-op BMI } \\
\text { (mean } \mathrm{Kg} / \mathrm{m}^{2} \pm \text { std dev) }\end{array}$ & $\begin{array}{l}\text { Type of } \\
\text { surgery }\end{array}$ & $\begin{array}{c}\text { Post-op } \\
\text { complications }\end{array}$ \\
\hline Olmi et al. (2019) ${ }^{[9]}$ & 1 & 38 & 43 & RYGB & None reported \\
\hline Frattini et al. $(2015)^{[10]}$ & 15 & 42 & 45 & Sleeve & None reported \\
\hline Di Furia et al. (2019) ${ }^{[11]}$ & 43 & 46.04 & 40.73 & Sleeve & 1 leak \\
\hline Ortega et al. (2018) $)^{[12]}$ & 86 & - & - & Sleeve & None reported \\
\hline Hagen et al. $(2019)^{[13]}$ & 95 & $43.9 \pm 11.1$ & $43.5 \pm 6.4$ & R-RYGB & None reported \\
\hline
\end{tabular}

Table 1: Characteristics of the included studies.

\section{Results:}

A total of 103 articles were identified during the search (Figure 1). After removal of duplicates, 75 papers were screened, of which 69 were not pertinent to the study and thus were excluded. Six eligible studies were initially identified, one of which was removed for noncompliance with the inclusion criteria. Ultimately, five studies were included for the analysis, all published after 2008. Our search found no comparative studies.

One of the studies was a case report[9]; the remaining four included studies [10-13] from prospectively maintained databases analyzed retrospectively (Table 1). A total of 240 patients were included in the studies. The majority of patients $(\mathrm{N}=144)$ underwent laparoscopic sleeve gastrectomy, another 95 underwent robotic Roux-en-Y gastric bypass (R-RYGB), and 1 underwent a laparoscopic RYGB combined with a Rossetti fundoplication. No demographic data were available for 86 patients, not having been reported in the study by Ortega et al.[12] No intraoperative complications were reported in any of the studies. Only one leak occurred postoperatively, in a patient who underwent a laparoscopic sleeve gastrectomy, as reported by Di Furia et al.[11] ICG was used intravenously to assess proper vascularization of the staple line in four of five studies. In the study by Hagen et al[13], however, ICG was used to assess intraoperative leaks and was administered via nasogastric tube, mixed with methylene blue dye and saline solution. No studies reported side effects following ICG administration.

Some of the studies included in our review described the effect of ICG administration on operative times, finding no significant increase. However, the lack of comparative studies prevented the drawing of any conclusions about the advantages of this technique over standard practice. Nor can any conclusion be drawn regarding the most appropriate dose and administration regimen of ICG, owing to a lack of information in some of the studies and the heterogeneity of application seen in the remainder.

\section{Discussion:}

At present, there is no gold standard method for conducting intraoperative assessment of tissue perfusion. Traditionally, visual evaluation and tactile testing of pulsating blood vessels have been most frequently adopted. The recent introduction of ICG in minimally invasive surgery has provided a potential solution to the problem, but its use is debated among the surgical community. The use of ICG in bariatric surgery was introduced only recently, with the aim of assessing adequate perfusion of the gastric tubule during sleeve gastrectomy and of bowel anastomoses in RYGB, so as to reduce leaks. However, in view of the low incidence of leaks in bariatric surgery[4], study of a large number of patients may be needed to detect any difference between standard practice and ICG, representing a potential obstacle to doing so. Consensus on optimal ICG dosage and timing of administration will also be needed. Notably, although some acute allergic reactions have been reported, the intravenous use of ICG is regarded as generally safe.[14]

Accordingly, we see revisional bariatric surgery as a potentially promising application of ICG because of the challenges provided by anatomical alterations and the need to achieve optimal perfusion in suboptimal tissues. A significant limitation of this technique is the current impossibility of providing objective quantitative assessment of fluorescence intensity, which would provide a more reliable way of demonstrating the value of this technology. In our opinion, this is an important need that should be addressed by novel studies.

Our study has several limitations. Data presented among the various studies were highly heterogeneous, preventing any comparison. All studies included were single-centered and adopted different techniques for ICG administration and imaging detection systems; indeed, some studies lacked such information altogether.

\section{Conclusion:}

ICG fluorescence is a widely adopted technique in multiple surgical specialties but has been introduced to bariatric surgery only recently. Its intravenous use allows the execution of real-time intraoperative angiography and assessment of tissue perfusion, thus potentially preventing leaks as well as perfusion-related complications. Currently no evidence suggests that its use can reduce postoperative complications in bariatric surgery. We think that ICG use may be a valuable aid to the surgeon for intraoperative real-time assessment of vascularization and tissue perfusion. More studies, particularly randomized controlled trials, comparing its application with standard vision are needed to investigate the potential advantages of ICG use in routine practice.

\section{Acknowledgements}

Not applicable.

\section{Contributors}

FMC, NDL conceptualized and designed the study, acquired and analysed data, interpreted the study results, drafted the manuscript. FMC, NDL critically 
revised the manuscript for important intellectual content.

\section{Funding}

No funding was received for this study.

\section{Competing interests}

No benefits in any form have been received or will be received from a commercial party related directly or indirectly to the subject of this article.

\section{Availability of data and materials}

Further information is available from the corresponding author on reasonable request.

\section{Ethics approval}

Not applicable.

\section{Provenance and peer review}

Not commissioned; externally peer reviewed.

\section{Open access}

This is an Open Access article distributed in accordance with the Creative Commons Attribution NonCommercial (CC BY-NC 4.0) license, which permits others to distribute, remix, adapt, build upon this work noncommercially, and license their derivative works on different terms, provided

the original work is properly cited and the use is non-commercial. See: http://creativecommons.org/ licenses/by-nc/4.0/

\section{References}

[1] Pareek M, Schauer PR, Kaplan LM, Leiter LA, Rubino F, Bhatt DL. Metabolic Surgery: Weight Loss, Diabetes, and Beyond. J Am Coll Cardiol. 2018;71:670-87.

[2] Aminian A, Brethauer SA, Kirwan JP, Kashyap SR, Burguera B, Schauer PR. How safe is metabolic/diabetes surgery? Diabetes Obes Metab. 2015;17:198-201.

[3] Arterburn D, Wellman R, Emiliano A, Smith SR, Odegaard AO, Murali S, et al. Comparative Effectiveness and Safety of Bariatric Procedures for Weight Loss: A PCORnet Cohort Study. Ann Intern Med. 2018;169:741-50

[4] Ghiassi S, Morton JM. Safety and Efficacy of Bariatric and Metabolic Surgery. Curr Obes Rep. 2020;9:159-64

[5] Purich K, Dang JT, Poonja A, Sun WYL, Bigam D, Birch D, et al. Intraoperative fluorescence imaging with indocyanine green in hepatic resection for malignancy: a systematic review and meta-analysis of diagnostic test accuracy studies. Surg Endosc. 2020.

[6] Alander JT, Kaartinen I, Laakso A, Patila T, Spillmann T, Tuchin $\mathrm{VV}$, et al. A review of indocyanine green fluorescent imaging in surgery. Int J Biomed Imaging. 2012;2012:940585.

[7] Moher D, Liberati A, Tetzlaff J, Altman DG, Group P. Preferred reporting items for systematic reviews and meta-analyses: the PRISMA statement. PLoS Med. 2009;6:e1000097.

[8] Slim K, Nini E, Forestier D, Kwiatkowski F, Panis Y, Chipponi J. Methodological index for non-randomized studies (minors): development and validation of a new instrument. ANZ J Surg. 2003;73:712-6.

[9] Olmi S, David G, Cesana G, Ciccarese F, Giorgi R, De Carli S, et al. Modified Sleeve Gastrectomy Combined with Laparoscopic Rossetti Fundoplication and Vascularization Assessment with Indocyanine Green. Obes Surg. 2019;29:3086-8.

[10] Frattini F, Lavazza M, Mangano A, Amico F, Rausei S, Rovera F, et al. Indocyanine green-enhanced fluorescence in laparoscopic sleeve gastrectomy. Obes Surg. 2015;25:949-50.

[11] Di Furia M, Romano L, Salvatorelli A, Brandolin D, Lomanto D, Cianca G, et al. Indocyanine Green Fluorescent Angiography During Laparoscopic Sleeve Gastrectomy: Preliminary Results. Obes Surg. 2019;29:3786-90.

[12] Ortega CB, Guerron AD, Yoo JS. The Use of Fluorescence Angiography During Laparoscopic Sleeve Gastrectomy. JSLS. 2018;22. [13] Hagen ME, Diaper J, Douissard J, Jung MK, Buehler L, Aldenkortt F, et al. Early Experience with Intraoperative Leak Test Using a Blend of Methylene Blue and Indocyanine Green During Robotic Gastric Bypass Surgery. Obes Surg. 2019;29:949-52.

[14] Speich R, Sasseli B, Hoffmann U, Neftel K. Letters and corrections. Ann Intern Med. 1988;109:345-6. 